\title{
Endodontic surgery prognostic factors
}

\author{
Abstracted from \\ von Arx T, Peñarrocha $M$, Jensen $S$. \\ Prognostic factors in apical surgery with root-end filling: \\ a meta-analysis. J Endod 2010; 36: 957-973. \\ Address for correspondence: Dr Thomas von Arx, Department of Oral Surgery and Stomatology, \\ School of Dental Medicine, University of Bern, Freiburgstrasse 7, CH-3010 Bern, Switzerland. \\ E-mail: thomas.vonarx@zmk.unibe.ch
}

\section{Question: What factors influence the prognosis of apical surgery with root-end filling?}

Data sources Medline, (PubMed) and the Cochrane databases together with hand searching of the following journals: Journal of Endodontics, International Endodontic Journal, Oral Surgery Oral Medicine Oral Pathology (name changed to Oral Surgery Oral Medicine Oral Pathology Oral Radiology and Endodontics in 1995), Endodontics and Dental Traumatology (name changed to Dental Traumatology in 2001), Journal of Oral and Maxillofacial Surgery, and International Journal of Oral and Maxillofacial Surgery.

Study selection Clinical studies evaluating apical surgery with placement of a root-end filling were included. Studies on apical surgery with orthograde root canal filling or about apicectomy alone without root-end filling were excluded, as were experimental and animal studies. Only studies with $\geq$ ten patients with a minimum six month follow-up period and clearly defined radiographic and clinical healing criteria, with healing reported for at least two categories of a specific prognostic factor were accepted. Studies reporting in English, German, French, Spanish, Italian, Portuguese and Scandinavian languages were included. All studies were assessed separately by two of the three authors, with disagreements resolved by discussion.

Data extraction and synthesis Prognostic factors were divided into patient related, tooth-related or treatment-related factors. The reported percentages of healed teeth were pooled per category. The statistical method of Mantel-Haenszel was applied to estimate the odds ratios and their $95 \%$ confidence intervals. Homogeneity was assessed using Woolf's test.

Results With regard to tooth-related factors, the following were identified as predictors of healing: absence of preoperative pain or signs, good density of the root canal filling and a periapical lesion size of $\leq 5 \mathrm{~mm}$. With regard to treatment-related factors, teeth treated with the use of an endoscope tended to have higher healed rates than teeth treated without the use of an endoscope.

Conclusions Although the clinician may be able to control treatmentrelated factors (by choosing a certain technique), patient- and toothrelated factors are usually beyond the surgeon's power. Nevertheless, patient and tooth-related factors should be considered as important prognostic determinants when planning or weighing apical surgery against treatment alternatives.

\section{Commentary}

Understanding the prognostic predictors for any type of treatment might be important in the process of decision-making between different treatment modalities. For apical surgery, such information can help the clinicians in appropriate case selection, increasing the likelihood of a favourable outcome. In an attempt to update the previous reviews, (narrative and systematic), this meta-analysis pooled the results of 38 published clinical studies to assess the potential predictors of healing in apical surgery with root-end filling.

The authors carefully designed and executed the search and clearly outlined the process of study selection. They extracted information from studies varying in design, from retrospective observational studies to randomized clinical trials. The primary aim to identify prognostic factors was explored through a series of forest plots for a number of factors, presented with raw data from eligible studies for readers to make their own impression regarding validity of the results. Tooth-related, patient-related and treatment-related factors were separately analysed and the data were presented. The rigour of the review notwithstanding, lapses in methodology ${ }^{1}$ require attention before the results can be applied in clinical practice.

The eligibility criteria of any clinical study with a minimum of ten subjects and a minimum follow-up period of six months are oversimplified. It is known that in general, 5-25\% of teeth classified as healed after one year may regress to periapical radiolucency or clinical symptoms when followed-up longer. ${ }^{4-6}$ Therefore, outcomes assessed in less than 3-4 years (reported in at least 14/35 included studies) should be considered to overestimate the potential for healing in the long-term. Sensitivity analyses accommodating duration of follow-up would have been beneficial.

It is surprising to note that studies of different levels of evidence (retrospective study to clinical trial) were all pooled together. No subgroup analysis based on type of study or level of evidence was reported, which undermines the validity of the pooled results, as bias inherent to different types of studies is not accounted for. Additionally, modern advancements in surgical endodontics, (use of the dental operative microscope to address anatomic intricacies and ultrasonic instruments for root-end cavity preparation to a depth of $3 \mathrm{~mm}$, with no or minimal bevel of the root-end resection), improve the precision and predictability of the surgical procedure in comparison with the traditional surgical procedure (root-end resection with a 45-degree bevel, cavity preparation with burs and inferior magnification and illumination facility). ${ }^{7}$ Comparing outcomes and prognosis with subgroup analysis could have shed more light on clinical practice. 
From the perspective of outcome assessment, the potential bias resulting from lumping the outcome categories 'complete healing' and 'incomplete healing' (scar) into one category of 'healed' was not explored in a sensitivity analysis. It should be noted that scar tissue (at one year), when adequately diagnosed, has been reported to occur in $<10 \%$ of teeth treated by apical surgery. ${ }^{8-10}$ There was no attempt to critically investigate the percentage of the 'incomplete healing' in the individual studies that may raise the possibility of misclassification bias.

The most important and critical component of a meta-analysis is the assessment of risk of bias in included studies. This can be accomplished using well designed criteria as suggested by Hayden et al. ${ }^{11}$ Sensitivity analyses excluding studies with high risk of bias (studies with a high rate of attrition) would have provided robustness to results.

Despite certain similarities between studies, significant clinical heterogeneity remained. At the same time, no measure of statistical heterogeneity has been explored. For example, the authors reported that for the definition of the length of root canal filling, two studies defined inadequate length as overfilled or $>2 \mathrm{~mm}$ underfilled, two studies as $>3 \mathrm{~mm}$ underfilled, and one study did not specify the definition. Combining data from such varied definitions can have a significant influence on the results.

Investigating the treatment-related factors (arguably under control of the surgeon), the authors found that resurgery cases, cases in which the root-end cavity preparation was done with a bur, cases where the root-end filling materials used were inferior to mineral trioxide aggregate (MTA) or cases in which no magnification was utilized had a poorer outcome. As mentioned above, the current approach to and advancement in the apical surgery procedure has made a considerable impact on the outcome. Therefore, it could be considered erroneous to pool the results of the traditional and modern approaches when examining the tooth- and patientrelated factors.

In conclusion, this meta-analysis summarized evidence of the prognostic factors for healing after apical surgery with root-end filling. While the paper pools the results of each of the prognostic factors, several methodological issues preclude one from making strong conclusions regarding the importance of individual factors. The approach, as deployed in the current review, should be considered very preliminary and exploratory. Since the search strategy termination date of this systematic review and meta-analysis, (2007), there have been several others, whose outcomes need also to be considered. A properly conducted systematic review with less biased methodology is needed in this area to be able to provide any implications of prognostic factors in appropriate case selection for apical surgery against treatment alternatives.

\section{Practice point}

- Understanding the patient-, treatment- and tooth-related prognostic predictors can help the clinicians in appropriate case selection for apical surgery against treatment alternatives. These factors should be considered in the process of decision making. Accordingly, an appropriate course of treatment or referral can be communicated with patients.

Amir Azarpazhooh,

Disciplines of Dental Public Health and Endodontics, Faculty of Dentistry, University of Toronto, Canada and

Prakesh S Shah,

Departments of Paediatrics and Health Policy, Management and Evaluation, Faculty of Medicine, University of Toronto, Canada

Drs Azarpazhooh and Shah thank Professor Shimon Friedman, Faculty of Dentistry, University of Toronto, for his thoughtful inputs on this commentary.

1. Moher D, Liberati A, Tetzlaff J, Altman DG. Preferred Reporting Items for Systematic Reviews and Meta-analyses: the PRISMA statement. Br Med J 2009; 339: b2535.

2. Rud J, Andreasen JO, Jensen JE. Radiographic criteria for the assessment of healing after endodontic surgery. Int / Oral Surg 1972; 1: 195-214.

3. Molven $\mathrm{O}$, Halse A, Grung B. Observer strategy and the radiographic classification of healing after endodontic surgery. Int J Oral Maxillofac Surg 1987; 16: 432-439.

4. Yazdi PM, Schou S, Jensen SS, Stoltze K, Kenrad B, Sewerin I. Dentine-bonded resin composite (Retroplast) for root-end filling: a prospective clinical and radiographic study with a mean follow-up period of 8 years. Int Endod / 2007; 40: 493-503.

5. Wesson CM, Gale TM. Molar apicectomy with amalgam root-end filling: results of a prospective study in two district general hospitals. Br Dent J 2003; 195: 707-714.

6. Kvist T, Reit C. Results of endodontic retreatment: a randomized clinical study comparing surgical and nonsurgical procedures. / Endod 1999; 25: 814-817.

7. Tsesis I, Faivishevsky V, Kfir A, Rosen E. Outcome of surgical endodontic treatment performed by a modern technique: a meta-analysis of the literature. J Endod 2009; 35: 1505-1511

8. Grung B, Molven O, Halse A. Periapical surgery in a Norwegian county hospital: follow-up findings of 477 teeth. J Endod 1990; 16: 411-417.

9. Jensen SS, Nattestad A, Egdø P, Sewerin I, Munksgaard EC, Schou S. A prospective, randomised, comparative clinical study of resin composite and glass ionomer cement for retrograde root filling. Clin Oral Investig 2002; 6: 236-243.

10. Altonen M, Mattila K. Follow-up study of apicoectomized molars. Int / Oral Surg 1976; 5: 33-40.

11. Hayden JA, Côté P, Bombardier C. Evaluation of the quality of prognosis studies in systematic reviews. Ann Intern Med. 2006; 144: 427-437.

Evidence-Based Dentistry (2011) 12, 12-13. doi:10.1038/sj.ebd.6400773 